\title{
Partial Androgen Insensitivity Syndrome
}

National Cancer Institute

\section{Source}

National Cancer Institute. Partial Androgen Insensitivity Syndrome. NCI Thesaurus. Code C120192.

A genetic disorder associated with a mutation in the AR gene, resulting in partial resistance to androgenic hormones. 